\title{
Fungi and the life of man
}

M. J. Carlile

Fungi, Man and his Environment. By R. C. Cook. Pp. 144. (Longman: London, 1977.) £6.75.

There are several good textbooks on the systematic, physiological and genetic aspects of the fungi. The present work is complementary to these, and provides a readable account of the ways in which fungi directly or indirectly affect the life of man. This is a particularly laudable endeavour, as the relevant literature is widely scattered, often in journals little read by and relatively inaccessible to most biologists. The author stresses that he has not attempted to produce a textbook or a uniform coverage of the field, but has written about topics that he has found interesting. The most important omissions, as the author admits, are the role of fungi in the preparation of traditional foods and drinks, and in the production of antibiotics. Apart from this, most of the economically important aspects of the fungi are covered.

The first chapter is a brief introduction to the fungi for non-mycologists. The next, "nutrients and niches", deals with the habitats of fungi in relation to nutrition and physiology, and the related topic of biodegradation, both beneficial, as in composts, and harmful, as in the various forms of biodeterioration such as timber decay and the spoilage of aviation fuel, food and stored products.

Plant disease is then discussed (chapter 3), with emphasis on recent catastrophic episodes such as Dutch Elm Disease in the United Kingdom and Jarrah Dieback in Australia. A chapter on symbiosis follows, with stress on lichens as indicators of atmospheric pollution and mycorrhiza as a major factor in the nutrition of trees and perhaps crop plants. Chapter 5 discusses fungal diseases of man and animals, including fish, and is followed by an account (chapter 6) of biological control, by fungi and of fungi. Chapter 7 is concerned with fungus-insect relationships: fungus gardens of ants, fungi as insect endosymbionts, and even insects as slaves of the fungi-scale insects extracting nutrients from plants on behalf of their fungus captors.

The chapter (8) on mycotoxins concentrates on the gruesome consequences of consuming one of the few really deadly mushrooms, Amanita phalloides, the effects of ergot and its derivatives, and the aflatoxin story. There is a short chapter (9) on the industrial production of microbial protein, and a longer one (10) on fungi and magic: fairy rings, luminous wood and hallucinogens. The book ends with a short bibliography of key references for each chapter, and an index.

There are a few errors, almost inevitable in a book covering so wide a field, but none that seriously detract from the value of the work. It is to be hoped that the publishers will refrain from continued use of such small print, which I found liable to cause eyestrain

except under very good light. The price seems rather high for such a slim volume, but is perhaps explained by the copious photographs and drawings, which are attractive and instructive, enhancing the value of the text. The author and publisher are to be congratulated on a pioneering work, which admirably demonstrates the practical importance of fungi, and which should be of value to both lecturers and students.

M. J. Carlile is Senior Lecturer in Microbiology in the Deparament of Biochemistry, Imperial College, London, UK.

\section{Aspects of high energy physics}

Deeper Pathways in High-Energy Physics. Edited by A. Perlmutter and L. F. Scott. Pp. 743. (Plenum: New York, 1977.) \$71.40.

THIS volume contains papers presented at the fourteenth annual Coral Gables Conference. The conference opened with a session on "Polarized Particles and Spin Effects in High Energy Physics". The recent development of a polarised proton beam at Argonne National Laboratory makes it possible to measure new properties of reaction amplitudes and the new data reported here are a challenge to all models of hadron reactions. The novel questions posed by such data are discussed in a useful review by Halzen.

The next session on "Supersymmetry and Supergravity" was devoted to important work on local supersymmetries carried out during 1976. One attraction of supersymmetries is that they link together particles of different spins in a single multiplet, in which there is a connection between the spin content and the internal symmetry content. However, despite the large amount of work which has been done on these mathematically elegant models "there are as yet no signals from experiment that nature is aware of our efforts, (so) we look for theoretical signals", to quote the article by Freedman. The possibility that the theory may be renormalisable is perhaps the most interesting signal. This is discussed in a particularly clear article by van Nieuwenhuizen and Grisaru. In supergravity (which only differs from Einstein's theory at short distances), the contributions to amplitudes due to exchanges of different particles in a multiplet are connected and tend to cancel in a remarkable way. This renders one- and two-loop amplitudes finite in certain models. If amplitudes turn out to be finite to all orders, supergravity would be the first renormalisable quantum theory of gravity, with which we could calculate to all orders in principle. Whather this happens and whether the models have anything to do with the real world are still open questions.

The remaining sessions were mainly on questions connected with what (unfortunately) is coming to be known as QFD-quantum flavourdynamics (the theory of weak-electromagnetic interactions, which couple to 'flavour' quantum numbers-charge, strangeness, charm, and so on) and QCD--quantum chromodynamics (a model in which strong interactions are generated by fields coupled to the 'colour' quantum number of hadrons). Despite changes in the data during the past year, the review of the status of QFD by Fritzsch and the discussion of how to search for heavy particles by Barnett are still valuable. The same is true of the paper by Cheng and $\mathrm{Li}$ on the decay $\mu \rightarrow \mathrm{e} \gamma$, although rumours that this decay had been observed turned out to be false. There is also a useful review by Bouchiat of the atomic physics experiments, which can constrain neutral couplings so strongly, and a good summary of ideas about charmed particle decays by Rosner. 